\title{
Passive Infrared Hemoencephalography (pIR HEG) for the Treatment of Migraine Without Aura
}

\author{
Alison K. Walker ${ }^{1 *}$ and Randall R. Lyle ${ }^{2}$ \\ ${ }^{1}$ Saint Mary's University, San Antonio, Texas, USA \\ ${ }^{2}$ Mount Mercy University, Cedar Rapids, lowa, USA
}

\begin{abstract}
Objective: To evaluate the impact of Passive Infrared Hemoencephalography (pIR HEG) in reducing headacherelated disability in adults with migraine without aura (MWOA). Methods: This quasi-experimental study enrolled 31 adults $(M$ age $=38.65$ years, range $=20-65$ years $)$ who met the International Classification of Headache Disorders (2nd ed.) criteria for migraine without aura (MWOA; IHS, 2004). All participants received the treatment. Participants completed a 10-week protocol of pIR HEG. Changes in headache impact were assessed at three points in time: baseline, after six treatment sessions, and after 10 treatment sessions. Outcome Measures: Headache Impact Test (HIT-6) and the Migraine Disability Assessment (MIDAS) questionnaire. Results: Significant reductions in HIT-6 scores were found between Pretest and Midtreatment, $p<.001$, and between Pretest and Posttest, $p<.001$. Significant reductions in MIDAS scores were found between Pretest and Posttest, $p<.001$. Results indicated MIDAS subscale A scores did not significantly change across the three time points. Significant reductions in MIDAS subscale B scores were found between Pretest and Midtreatment, $p<.001$, and between Pretest and Posttest, $p<.001$. In this study, pIR HEG appeared to be effective by the end of treatment in reducing the impact of headache-related disability among the participants.
\end{abstract}

Keywords: migraine without aura; Passive Infrared Hemoencephalography; Headache Impact Test-6; Migraine Disability Assessment questionnaire; migraine-related disability

Citation: Walker, A. K., \& Lyle, R. R. (2016). Passive Infrared Hemoencephalography (pIR HEG) for the Treatment of Migraine Without Aura. NeuroRegulation, 3(2), 78-91. http://dx.doi.org/10.15540/nr.3.2.78

*Address correspondence to: Alison K. Walker, LMFT, Deep Eddy Psychotherapy, PLLC, 508 Deep Eddy Avenue, Austin, TX, 78703, USA. Email: alisonkathleenwalker@gmail.com

Copyright: @ 2016. Walker and Lyle. This is an Open Access article distributed under the terms of the Creative Commons Attribution License (CC-BY).

Edited by:

John Davis, PhD, McMaster University, Hamilton, Ontario, Canada

Reviewed by:

John Davis, PhD, McMaster University, Hamilton, Ontario, Canada Nancy L. Wigton, PhD, Grand Canyon University, Phoenix, Arizona, USA

\section{Introduction}

Migraine is a highly prevalent, episodic, chronic pain condition characterized by disabling attacks and impaired functioning between attacks (Buse, Rupnow, \& Lipton, 2009; Dahlöf \& Solomon, 2006; Diamond et al., 2006; International Headache Society [IHS], 2004; Lipton, Stewart, Sawyer, \& Edmeads, 2001). Migraine is a primary headache disorder (i.e., headache that lacks a clear diagnostic or biochemical marker; IHS, 2004) that produces substantial physical suffering and impairs functioning. In addition, migraine can place an enormous economic burden on individual sufferers, their families, and on society. Acute migraine attacks are generally characterized by headache of a throbbing or pulsating quality, photophobia (increased sensitivity to light), phonophobia (increased sensitivity to sound), and nausea, vomiting, or both (IHS, 2004). Pain-free periods are often marked by hyper-vigilance, affective distress, diminished energy levels, increased anxiety and fear, and reduced willingness or capacity to participate in work, family, and social activities (Buse et al., 2009).

There is currently no cure for migraine. At best, current treatments can reduce the frequency and severity of acute migraine attacks. Oftentimes, outcomes are optimized when pharmacologic treatments are used in conjunction with nonpharmacologic treatments (Blumenfeld \& Tischio, 2003; Buse et al., 2009; Harpole et al., 2003; Lemstra, Stewart, \& Olszynski, 2002; Mathew \& Tfelt-Hansen, 2006). Although a multidisciplinary approach to treatment often produces the best 
outcomes, medications are most commonly used in the treatment of migraine. There has been a proliferation of pharmacologic treatments within the past two decades. Although medications can reduce migraine symptoms, they provide only a partial benefit. Current medications provide some benefit for 50 to $60 \%$ of people with migraine (Berg \& Ramadan, 2006, p. 35). In addition, pharmacologic therapies are not an option for people with heart disease or who have had a stroke (Tfelt-Hansen, 2006). Due to these and other limitations associated with pharmacologic treatments, many do not receive effective relief.

Nonpharmacologic interventions provide an alternative treatment approach for the treatment and management of the symptoms and the overall impact of migraine. Nonpharmacologic interventions have demonstrated efficacy in reducing the severity and the frequency of migraine (Penzien et al., 2005).

Outcomes for complex disorders like migraine are often optimized when treatment incorporates pharmacologic and nonpharmacologic therapies (Penzien et al., 2005). Pharmacologic treatments are designed to reduce or manage the acute symptoms associated with a migraine attack. Nonpharmacologic treatments are generally designed to address the underlying pathophysiology of migraine. Continued development and evaluation of novel therapies is essential to identify opportunities to reduce the burden of the disease and improve the quality and effectiveness of treatment strategies for migraine.

One relatively new intervention that warrants further examination is Passive Infrared Hemoencephalogy ( $p I R$ HEG); which is a nonpharmacologic intervention, developed specifically for the treatment of migraine disorders, that has shown promise in reducing migraine impact (Carmen, 2004). While pIR HEG has been used clinically for over a decade, only one case series (Carmen, 2004) has evaluated the impact of pIR HEG for individuals with migraine disorders. Based on the results of this study, Carmen suggested that pIR HEG may be a useful intervention for reducing the severity of acute attacks of migraine. Although preliminary results appear promising, further studies are needed to determine the effects of pIR HEG in the treatment of migraine. This study is designed to expand on the case series by Carmen (2004) and to contribute to the literature regarding nonpharmacologic interventions for the treatment of migraine.
The primary objective of this study was to evaluate the efficacy of pIR HEG in reducing headacherelated disability, attack frequency, and attack severity in a sample of adults with a migraine subtype, migraine without aura (MWOA), based on ICHD diagnostic criteria (see Table 1). Specifically, this study assessed headache-related disability, attack frequency, and attack severity using pretest and posttest measures. Two instruments designed to quantify the impact of headache-related disability, the Headache Impact Test (HIT-6; Nachit-Ouinekh et al., 2005) and the Migraine Disability Assessment (MIDAS; Stewart, Lipton, Whyte, et al., 1999) questionnaire, were used to assess the effectiveness of the intervention. The HIT-6 assesses the global impact of headache-related disability (Dahlöf \& Solomon, 2006; Kosinski et al., 2003; Nachit-Ouinekh et al., 2005). The MIDAS questionnaire assesses the functional impact of headache-related disability and includes two subscales that assess the frequency and severity of migraine, respectively (Stewart, Lipton, \& Kolodner, 2003; Stewart, Lipton, Kolodner, Liberman, \& Sawyer, 1999). When used together, these instruments provide a comprehensive picture of headache impact.

\section{Migraine Headache}

Migraine is a chronic and potentially progressive pain disorder that inflicts a very high burden on individual sufferers, their families, and on society (IHS, 2004). Migraine-related disability produces substantial impairment during and between attacks. Attack-related disability ranges from temporary mild impairment to complete incapacitation for days and sometimes weeks. Impairment during pain-free periods produces emotional distress and reduced functioning and productivity.

The degree of disability that migraine causes depends largely on the frequency, duration, and severity of acute attacks. The rate of attack occurrence can range from one to two attacks annually to attacks daily (IHS, 2004). Migraine prevalence typically peaks between 25 and 55 years of age, the most productive years of the life span (Abramson, Hopp, \& Epstein, 1980; Bille, 1981; Dalsgaard-Nielsen, Engberg-Pedersen, \& Holm, 1970; Lipton et al., 2007, 2002; Lipton, Stewart, Diamond, S., Diamond, M. L., \& Reed, 2001; Nikiforow, 1981; Patel et al., 2004; Sillanpää \& Anttila, 1996; Stang \& Osterhaus, 1993; Steiner, Scher, Stewart, Kolodner, Liberman, \& Lipton, 2003; Stewart, Shechter, \& Rasmussen, 1994; Stewart, Lipton, Celentano, \& Reed, 1992; Stovner \& Scher, 2006). Over time, migraine-related disability may 
affect the physical and emotional well-being of individual sufferers, diminish health-related quality of life, increase healthcare costs, and reduce the individual's willingness or capacity to participate in work, family, and social activities (Dahlöf \& Solomon, 2006; Diamond et al., 2007; IHS, 2004).

To minimize the pain and disability migraine produces, most migraine sufferers take various combinations of medications that address symptoms associated with their migraines. Although medications have provided relief for many, they provide only partial benefit. Limiting factors associated with medications for the treatment of migraine include inadequate response, adverse events such as headache recurrence and addiction, potential drug interactions, formulary restrictions, contraindications, high cost, and patient preference for a nonpharmacologic treatment (Lipton, Stewart, Diamond, S., Diamond, M. L., \& Reed, 2001; Mathew \& Tfelt-Hansen, 2006).

Limitations associated with symptomatic medications combined with the substantial social and economic consequences of migraine underscore the need to improve current approaches for managing migraine. Developing and evaluating novel treatments that are cost-effective and can be shown to reduce the impact of disability associated with migraine may be one of the best ways to optimize outcomes.

Nonpharmacologic therapies may reduce attackrelated disability and the overall impact of migraine. In addition, nonpharmacologic therapies have been shown to enhance personal control of headache, reduce treatment costs, and sustain long-term improvements. Nonpharmacologic therapies may be particularly well-suited for individuals who are unable or unwilling to take drugs (e.g., those who are pregnant, nursing, or planning to become pregnant); who have an insufficient response to pharmacologic therapies, poor tolerance for medications, or with a history of frequent or excessive use of analgesics or acute medications (McGrath, Penzien, \& Rains, 2006).
Despite empirical evidence demonstrating the effectiveness of nonpharmacologic therapies in producing clinically meaningful reductions in headache impact, they are rarely integrated into treatment strategies for managing migraine (McGrath et al., 2006). A variety of factors contribute to the underutilization of nonpharmacologic therapies in migraine management. As previously discussed, the paucity of research on nonpharmacologic therapies for the treatment of migraine has led many healthcare providers to overlook the value and costeffectiveness of these therapies in migraine management.

Headache classification and migraines. Major headache groups are subdivided into types, subtypes, and subforms (IHS, 2004). For example, migraine is a primary headache that consists of one group (1. Migraine), one type (migraine), six subtypes (1.1 Migraine without aura, 1.2 Migraine with aura, 1.3 Childhood periodic syndromes that are commonly precursors of migraine, 1.4 Retinal migraine, 1.5 Complications of migraine, 1.6 Probable migraine), and 17 subforms (1.2.1 Typical aura with migraine headache, 1.2.2 Typical aura with non-migraine headache, 1.2.3 Familial hemiplegic migraine et al.; IHS, 2004).

Within the literature headache disorders are identified with varying degrees of specificity. For instance, migraine is commonly used as an umbrella term to encompass more than one type of migraine. At times, the precise migraine syndromes are identified, but oftentimes they are not. For this reason, this paper identified headache disorders with the degree of specificity that was consistent with the literature.

In addition, most studies have examined MWOA and migraine with aura (MWA) together, with the exception of studies on migraine mechanisms and genetic studies (Olesen \& Goadsby, 2006). Considerably less research has examined MWOA individually. Consequently, it was not possible to review all aspects of MWOA separately. 


\section{Table 1}

ICHD-Il diagnostic criteria for MWOA

A. At least five attacks fulfilling criteria $B-D$

B. Headache attacks lasting 4-72 hours (untreated or unsuccessfully treated)

C. Headache has at least two of the following characteristics:

1. Unilateral location

2. Pulsating quality

3. Moderate or severe pain intensity

4. Aggravation by or causing avoidance of routine physical activity (e.g. walking or climbing stairs)

D. During headache at least one of the following:

1. Nausea and/or vomiting

2. Photophobia and phonophobia

E. Not attributed to another disorder

Source: IHS, 2004.

Phases of a migraine attack. Four distinct phases of a migraine attack have been identified: premonitory symptoms, sensory phase, pain phase, and postdrome (Zagami \& Bahra, 2006).

The first phase of a migraine attack is characterized by premonitory symptoms. Premonitory symptoms are symptoms that indicate disease onset. Premonitory symptoms for migraine are physiological and emotional. Typical physiological premonitory symptoms include frequent urination, water retention, constipation, nausea, repetitive yawning, slurred speech, pallor, visceral dilation, blurred vision, neck stiffness, muscle tension, photophobia, and phonophobia. Typical emotional premonitory symptoms include cravings for particular foods, increased appetite, fatigue, insomnia, hypoactivity, hyperactivity, anxiety, euphoria, irritability, difficulty concentrating, depression, and other less typical symptoms. Premonitory symptoms occur in various combinations and typically precede the attack by 2 to 48 hours (IHS, 2004; Parsons, 2006; Sacks, 1992; Zagami \& Bahra, 2006).

The second phase of a migraine attack is the sensory phase. The sensory phase is characterized by neurological symptoms called auras (Cutrer \& Olesen, 2006). Auras are fully reversible "focal neurological symptoms that usually precede and sometimes accompany the headache" (IHS, 2004, p. 24). Migraine auras include visual, sensory, language, and motor disturbances. The most common type of migraine aura is visual aura (IHS, 2004). Visual auras are "the manifestations of focal and cerebral dysfunction" (IHS, 2004). Visual migraine auras include scotomas ("a spot in the visual field in which vision is absent or distorted"; Merriam-Webster, 2005, p. 1114), diffuse blurring, distortions, hallucinations, scintillations (bright visual hallucinations that fluctuate in intensity; IHS, 2004), loss of vision, flickering lights, and spots or lines (Cutrer \& Olesen, 2006; IHS, 2004). The following types of aura are experienced in descending order of frequency: sensory, language, and motor disturbances. Sensory symptoms may include paresthesia ("a sensation of pricking, tingling, or creeping on the skin that has no objective cause"; Merriam-Webster, 2005, p. 901), tingling, numbness, and a loss of awareness of a body part (Cutrer \& Olesen, 2006; IHS, 2004). Language symptoms may include aphasia ("loss or impairment of the power to use or comprehend words"; MerriamWebster, 2005, p. 57) and dysarthria ("difficulty in articulating words due to disease in the central nervous system"; Merriam-Webster, 2005, p. 389). Motor symptoms may include motor weakness and paresis ("slight or partial paralysis"; MerriamWebster, 2005, p. 901). Migraine auras develop gradually, over 5 to 20 minutes, and last for less than 60 minutes (IHS, 2004).

The third phase of a migraine attack is the pain phase. The pain phase is usually the most painful and debilitating part of a migraine attack. Prior to the onset of the pain phase individuals often experience a state of dread or depression. Headache is generally considered the hallmark characteristic of the pain phase. The quality of headache is commonly described as stabbing, pressing, bursting, throbbing, pulsating, icepick pains or jabs and stabs, and "momentary sharp shooting pains in various parts of the head, including the eye" (Zagami \& Bahra, 2006, p. 402). Location of headache is usually hemicranial (i.e., located on either the right or left side of the head, does not cross the midline; IHS, 2004). Location of headache may also be bilateral (i.e., frontal temporal or occipital only; IHS, 2004) or generalized (i.e., affecting the whole head). Headache is a symptom of all forms of migraine with two exceptions: typical aura with nonmigraine headache and typical aura without headache (Cutrer \& Olesen, 2006; IHS, 2004). Although headache is generally considered the hallmark characteristic of the pain phase, it is never the sole symptom. Accompanying symptoms may include photophobia, phonophobia, abdominal pain, vomiting, tenderness or pain in or behind the eyeball, bloodshot eyes, sinus discomfort, fever, constipation, diarrhea, increased urination, swollen face, and cold hands or feet. Accompanying symptoms occur in various combinations and may 
change from one attack to the next. The pain phase usually lasts for several hours to several days and sometimes weeks (IHS, 2004; Olesen \& Dodick, 2006; Sacks, 1992; Zagami \& Bahra, 2006).

The final phase of a migraine attack is postdrome. Postdrome is commonly referred to as the "migraine hangover" because individuals often remain symptomatic even after the headache and accompanying symptoms have subsided. Typical postdrome symptoms include mood changes, fatigue, muscular weakness, and reduced appetite. Resolution of these and other less typical postdrome symptoms is usually gradual and often achieved by taking various combinations of symptomatic medication, vomiting, bed rest, sleep, and retreating to a dark and quiet room. Postdrome usually lasts for several hours to several days (IHS, 2004; Zagami \& Bahra, 2006).

Hemoencephalography. Hemoencephalography (HEG) is a form of neurofeedback (NF) that was invented by Hershel Toomim in 1995 and makes use of light in the range of red to infrared wavelengths to access and monitor blood flow changes in the prefrontal cortex (Toomim \& Carmen, 2009). Infrared emissions are electromagnetic radiation with wavelengths longer than visible light but shorter than radio waves. Any object that has a temperature above absolute zero emits infrared emissions. Emissions from tissue in the brain "reflect the level of metabolic activity which, in turn, is responsive to oxygen levels, neurotransmitter levels, blood flow, and other variables" (Freides \& Aberbach, 2005, p. 55). The primary objective of HEG NF is to train increases in neural activity in the prefrontal cortex by "inducing increases in cerebral blood flow" (Toomim \& Carmen, 2009, p. 190).

HEG NF is typically provided to sites in the prefrontal cortex, commonly referred to as the executive center of the brain, which consists of Brodmann Areas 9, $10,11,12,44,45,46$, and 47 . The primary function of the prefrontal cortex is to regulate complex cognitive, emotional, and behavioral functioning (Pliszka, 2003). HEG NF is typically provided for brain activity in the region of Braodmann Area 10 or adjacent areas.

HEG depends on changes in characteristics of blood (oxygen, temperature) that are correlated with changes in brain activity and metabolism. The color and temperature of cerebral blood flow provides information about neuronal activity. The brain requires oxygen and glucose to perform activities. Metabolically active areas of the brain require more oxygen and glucose. As oxygenated blood infuses the tissue based on use-dependent demand, the brain gets redder, and as the glucose and other bloodborne nutrients are metabolized it gets warmer. Consequently, metabolically active areas of the brain are redder and warmer, whereas metabolically inactive areas of the brain are purple and cooler (Toomim \& Carmen, 2009). HEG measurements of oxygen and temperature can be used to operantly shape increased brain activity.

HEG is designed to train increases in the brain's ability to regulate its physiological activity so that maladaptive patterns of brain function can be better regulated and result in reduced symptoms and enhanced performance. The primary objective of HEG training is to improve the control and management of symptoms by perturbing older more stable patterns of physiologic function.

HEG has demonstrated efficacy in improving treatment outcomes in a plethora of neurologic conditions. These include attentiondeficit/hyperactivity disorder (ADHD), autistic spectrum disorders, bipolar disorder, traumatic brain injury (TBI), age-related memory loss, migraine and other headaches, epilepsy, Tourette's disorder, obsessive compulsive disorder (OCD), stroke, depression, schizophrenia, and toxic encephalopathy (Toomim \& Carmen, 2009). The central mechanisms underlying the conditions noted above include malfunctioning brain modules and networks, and difficulties with arousal and inhibition. Specifically, these conditions are hypothesized to be caused by an excessive response by the brain in terms of the rate and magnitude to relatively minor stimuli (Toomim \& Carmen, 2009).

Research Questions. This study examined the following research questions:

1) Do participants experience a statistically significant reduction in the global impact of headache-related disability, as evidenced by the reduction of HIT-6 scores after six and 10 treatment sessions with pIR HEG?

2) Do participants experience a statistically significant reduction in the functional impact of headache-related disability, as evidenced by the reduction of MIDAS questionnaire scores after six and 10 treatment sessions with pIR HEG?

3) Do participants experience a statistically significant reduction in the frequency of attacks of MWOA, as evidenced by the reduction of MIDAS subscale A scores after 
six and 10 treatment sessions with pIR HEG?

4) Do participants experience a statistically significant reduction in the severity of attacks of MWOA, as evidenced by the reduction of MIDAS subscale $B$ scores after six and 10 treatment sessions with pIR HEG?

\section{Method}

This study implemented a quasi-experimental research design, which involves nonrandom assignment of participants to the treatment and does not manipulate the presence or absences of the independent variable (i.e., HEG NF). According to Heppner, Kivlighan, and Wampold (1999) quasiexperimental designs "can be especially useful in the evaluation of new and innovative programs" ( $p$. 172) and are appropriate in clinical settings when withholding treatment from participants (placebo) is unethical. The same measures were employed at three time points: baseline (Pretest), after the sixth treatment session (Midtreatment), and after the tenth treatment session (Posttest); thereby utilizing a repeated-measures research design. This study was approved by the St. Mary's university Institutional Review Board.

\section{Participants}

Adults (28 women, 3 men, $M$ age $=38.65$ years, range: 20-65 years) who met ICHD-II (IHS, 2004) diagnostic criteria for MWOA were recruited from three cities in Texas: San Antonio, Schertz, and Austin. Participants were recruited through flyers posted at counseling centers, an online advertisement, and referrals from healthcare professionals (physicians, neurologists, and counselors). Eligible participants were 18 years and older, fulfilled ICHD-II diagnostic criteria for MWOA, and were under the continual care of a physician or neurologist for MWOA throughout the study. Individuals who had received a previous diagnosis of a secondary headache disorder, evidence of medication overuse or abuse, consumption of illegal drugs or current drug use, and concurrent participation in other research projects were excluded.

In addition, participants had to have a baseline HIT6 score of $\geq 56$, indicating that migraines were having a substantial to very severe headache impact. All participants were exposed to the intervention. Table 2 depicts mean demographic information regarding the entire sample population.

\section{Table 2}

Demographic Characteristics of Participants

\begin{tabular}{|c|c|c|}
\hline Characteristic & $n$ & $\%$ \\
\hline \multicolumn{3}{|l|}{ Self-identity } \\
\hline Caucasian & 23 & 74 \\
\hline African American & 1 & 3 \\
\hline Hispanic or Latino & 5 & 16 \\
\hline Other & 2 & 6 \\
\hline \multicolumn{3}{|l|}{ Relationship status } \\
\hline Single, never married & 8 & 25 \\
\hline In a committed relationship & 2 & 6 \\
\hline Cohabitating & 1 & 3 \\
\hline Married & 16 & 51 \\
\hline Divorced but remarried & 2 & 6 \\
\hline Divorced & 1 & 3 \\
\hline Engaged & 1 & 3 \\
\hline \multicolumn{3}{|l|}{ Education level completed } \\
\hline Some college & 5 & 16 \\
\hline Associate degree & 1 & 3 \\
\hline Bachelor's degree & 14 & 45 \\
\hline Master's degree & 9 & 29 \\
\hline $\mathrm{PhD}$ & 1 & 3 \\
\hline J.D. & 1 & 3 \\
\hline \multicolumn{3}{|l|}{ Family history of migraine } \\
\hline Yes & 23 & 74 \\
\hline No & 2 & 6 \\
\hline Unsure & 6 & 19 \\
\hline
\end{tabular}

Note. $N=31$.

Table 3 depicts Means and Standard Deviations for Age of Onset and Years Lived with MWOA. 
Table 3

Means and Standard Deviations for Age of Onset and Years Lived with MWOA

\begin{tabular}{|c|c|c|c|c|}
\hline \multirow[b]{2}{*}{ Variable } & \multirow[b]{2}{*}{$M$} & \multirow[b]{2}{*}{$S D$} & \multicolumn{2}{|c|}{$\underline{95 \% \mathrm{Cl}}$} \\
\hline & & & LL & UL \\
\hline Age of onset of MWOA & 16.42 & 7.65 & 13.62 & 19.22 \\
\hline Years lived with MWOA & 22.90 & 12.96 & 18.15 & 27.66 \\
\hline
\end{tabular}

Note. $N=31$. $\mathrm{Cl}=$ confidence interval; $\mathrm{LL}=$ lower limit; $U L=$ upper limit.

\section{Role of the Researcher}

The principal investigator administered the demographic questionnaire, headache instruments, and the treatment, as well as oversaw all aspects of the study. The principal investigator had previously received formal training in neurofeedback from a clinician with board certification in neurofeedback from the Biofeedback Certification International Alliance $(\mathrm{BCI})$. In addition, the principal investigator received supervision from Jeffrey Carmen, who was the subject matter expert on pIR HEG, prior to and during the study.

\section{Measures}

HIT-6. The HIT-6 was developed by an international team of headache experts and psychometricians and is intended to be used by persons 18 years and older. The HIT-6 is among the most widely used instruments for assessing the impact of headaches and headache treatments and is useful for screening and monitoring changes in headache impact over time. The HIT-6 is a paper-form survey that measures the global impact of headache-related disability on the individual sufferer's life. Specifically, the HIT-6 measures the impact of headaches on the level of functioning and the well-being of the respondent. The HIT- 6 has been used in scholarly research, clinical research and practice, disease management, population monitoring, and risk assessment (Dahlöf \& Solomon, 2006; Kosinski et al., 2003; Nachit-Ouinekh et al., 2005).

The HIT-6 is a six-item self-administered questionnaire that assesses six domains of headache impact: cognitive functioning, psychological distress, pain, role-functioning, social functioning, and vitality. Response options for each item are never, rarely, sometimes, very often, and always. Response options are scored as: Never = 6; Rarely = 8; Sometimes = 10; Very often = 11; and Always = 13 (Kosinski et al., 2003). Recall period for all items is the previous 4 weeks (Bjorner, Kosinski, \& Ware, 2003b; Gandek, Alacoque, Uzun, Andrew-Hobbs, \& Davis, 2003; Kosinski et al., 2003;
Nachit-Ouinekh et al., 2005). The HIT-6 score is derived as the sum of the six items with minimum and maximum possible values of 36 and 78 , respectively. Higher scores are indicative of greater headache impact. HIT-6 scores are rated on a 4point grading system. The 4-point grading system for the HIT-6 total score is as follows: grade 1: Little to no impact (score $\leq 49$ ); grade 2: Some impact (score = 50-55); grade 3: Substantial impact (score = 56-59); and grade 4: Very severe impact (score $\geq$ $60)$.

The HIT-6 has demonstrated evidence of reliability and validity. The internal consistency and test-retest reliability estimates were .89 and .80 , respectively (Kosinski et al., 2003).

MIDAS questionnaire. The MIDAS questionnaire was based in part on input from an expert advisory committee and the Headache Impact Questionnaire (Stewart, Lipton, \& Kolodner, 2003; Stewart, Lipton, Kolodner, et al., 1999). Among the most widely used instruments for assessing the impact of headache-related disability, the MIDAS questionnaire was used to measure the functional impact of headache-related disability. The MIDAS questionnaire assesses lost time because of headache in three domains of activity: work for pay or school, household work or chores, and family, social, and leisure activities (Stewart, Lipton, Kolodner, et al., 1999; Stewart, Lipton, Whyte, et al., 1999).

The MIDAS questionnaire is a five-item selfadministered questionnaire that assesses time lost for headache in work for pay or school, household work or chores, and family, social, and leisure activities. Responses for each item are scaled in units of the number of days missed and the number of days activity was reduced by $50 \%$ or more for headache. The first three items ask respondents about the number of days they missed for headache in work for pay or school; household work or chores; and family, social, and leisure activities. The next 
two items ask respondents about the number of days their productivity was reduced by $50 \%$ or more for headache in work for pay or school and household work or chores (Bjorner, Kosinski, \& Ware, 2003a; Stewart, Lipton, \& Kolodner, 2003; Stewart, Lipton, Whyte, et al., 1999).

The MIDAS score is derived as the sum of the five items, with minimum and maximum possible values of 0 and 91 , respectively. Higher scores are indicative of greater headache impact. MIDAS scores are rated on a 4-point grading system. The 4-point grading system for the MIDAS score is as follows: grade I: Little or no disability (score $=0-5$ ); grade II: Mild disability (score $=6-10$ ); grade III: Moderate disability (score $=11-20$ ); and grade IV: Severe disability (score $\geq 21$ ).

Two additional items, MIDAS subscale questions $A$ and $B$, assess the frequency and the severity of headache attacks, respectively, but these items do not contribute to the MIDAS score. The frequency of attacks is measured in units of the number of days that headache was present. The severity of attacks is ranked on a 10-point scale ranging from 0 (no pain at all) to 10 (pain as bad as it can be). Recall period for all items is the previous 3 months (Dahlöf \& Solomon, 2006; Stewart, Lipton, \& Kolodner, 2003; Stewart, Lipton, Whyte, et al., 1999).

The MIDAS questionnaire has demonstrated evidence of reliability and validity (Stewart, Lipton, Kolodner, et al., 1999; Stewart, Lipton, Whyte, et al., 1999). For the individual MIDAS items, the testretest Spearman correlation coefficient ranged from .67 to $.73(p<.001$; Stewart, Lipton, Kolodner, et al., 1999); the test-retest Pearson correlation coefficient ranged from .60 to $.70(p<.001)$. For the MIDAS score the test-retest correlations were high (Spearman correlation coefficient $=.84$; Pearson correlation coefficient $=.75, p<.001$; Stewart, Lipton, Kolodner, et al., 1999). Cronbach's alpha was high $(\alpha=.83, p<.001$; Stewart, Lipton, Kolodner, et al., 1999).

Both the HIT-6 and the MIDAS questionnaire are brief, comprehensive, and easy to interpret while providing meaningful scores. In addition, both instruments are sensitive enough to reflect changes in headache impact over time and may be used as outcome measures to monitor the effectiveness of a treatment (Dahlöf \& Solomon, 2006; Stewart, Lipton, Kolodner, et al., 1999; Stewart, Lipton, Kolodner, Sawyer, et al., 2000). Although the HIT-6 and the MIDAS questionnaire quantify the impact of headache-related disability, these instruments differ in content and design. First, the HIT-6 assesses multiple dimensions of headache impact (global disability), whereas the MIDAS questionnaire assesses one dimension of headache impact (functional disability). Second, the HIT-6 includes information on fatigue, cognition, and mental distress; the MIDAS questionnaire includes information on attack frequency and attack severity. Third, these instruments differ in response format (5point response scale vs. number of missed days due to headache, respectively) and the time interval assessed (4 weeks vs. 3 months, respectively; Bjorner et al., 2003a; Magnoux, Freeman, \& Zlotnik, 2007).

Finally, correlations between the HIT-6 and the MIDAS questionnaire ranged between .42 and .44 , suggesting that these instruments are weakly correlated (assess different dimensions of headache-related disability), and therefore should not be used interchangeably (Magnoux et al., 2007). The rationale for using these instruments together was to gain a more comprehensive picture of headache-related disability.

Demographic questionnaire. The demographic questionnaire was designed to elicit information about participants' age, gender, ethnicity, relationship status, occupation, education level, family history, medical history, age of onset for MWOA, and the number of years lived with MWOA. An additional item asks respondents about the manner in which he or she learned about the study. The demographic questionnaire was created by the principal investigator for the purpose of this research project.

\section{Materials}

This study used a notebook computer (HP Pavillion dv7.1245dx), the EZPIR system (Jeff Carmen; Manlius, NY), and DVDs. The EZPIR system has two major components: the headset and encoder hardware, and BioEra software (Proatech LLC, www.proatech.com; version 2.3.109). The headset is a small black box that houses dual overlapping sensors. The sensors detect infrared radiation within the 7 to 14 micron range and have a rectangular field of view of approximately 1.5 inches (in height) by 2 inches (in diameter) with a response speed of $30 \mathrm{~ms}$ (Carmen, 2004; Toomim \& Carmen, 2009). As shown in Figure 1, the headset is attached to an adjustable elastic headband which is secured around the top of the individual's head and the headset is positioned at Fpz (i.e., the center of the forehead). The headset connects to an encoder. The encoder is a rectangular box that converts the 
acquired signal (analog signal) into a digital USB signal, and then sends the digitized signal to the computer where it is processed by the BioEra software. To ensure the integrity of the pIR HEG system and to avoid potential interference with the software, following the installation of the software, the wireless assistant was disabled and no additional programs were installed on the notebook computer.

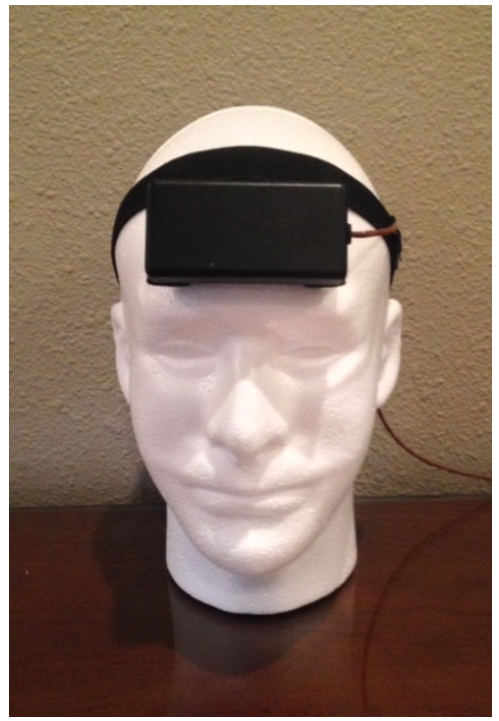

Figure 1. pIR HEG Headset.

\section{Procedure}

Upon referral, potential participants underwent a pre-screening to assess their initial eligibility and their interest in the study. At this time, the principal investigator answered any questions about the study protocols. This screening took place over the telephone and took approximately 10 minutes to complete. Those who did not qualify were excluded, thanked for their time, and given referral information for alternative treatments for MWOA.

Potential participants scheduled a meeting with the principal investigator and were asked to plan on spending 1 hour. At the initial meeting, the principal investigator provided a cover letter, discussed confidentiality, and answered any remaining questions about the study protocols. Informed consent was obtained from each participant in accordance with the approval from St. Mary's Institutional Review Board.

After informed consent was obtained, participants completed the HIT-6 to determine whether they qualified for continued participation in this research project. Participants needed a baseline HIT-6 score of $\geq 56$ to be eligible for continued participation. Participants with a baseline HIT- 6 score of $\leq 55$ were excluded. The HIT-6 took approximately 5 minutes to complete. Qualified participants completed a demographic questionnaire and the MIDAS questionnaire, and then began treatment. The MIDAS questionnaire and the demographic questionnaire took approximately 10 minutes to complete.

All participants received the treatment. The treatment consisted of administration pIR HEG. The treatment was conducted in accordance with clinical practice guidelines for administering pIR HEG in individuals with migraine. The treatment consisted of 10 sessions delivered, on average, 1 week apart. Once a week was the planned session frequency, however, due to scheduling conflicts (e.g., child care and work obligations) sessions were sometimes less frequent than once a week. On average, the intersession interval was 1.5 weeks. Sessions took place at one of three clinical settings: the Family Life Center at St. Mary's University, the Schertz Family Support Center, or the principal investigator's private practice in Austin, TX. For all participants, the first three sessions took place in the morning. The remaining seven sessions took place at various times throughout the day. The location and the time of the sessions were scheduled at the convenience of the participant. The duration of each treatment session was approximately 1 hour.

Part of the treatment involved the participants watching a movie on the screen of the notebook computer. Participants were given the option to bring in a DVD or to select a DVD from the principal investigator's personal collection. Participants were instructed to select movies that would engage their emotions and draw them into the plot so that they lost awareness of self, the room, etc., and to avoid movies that elicit shock or fear.

Each session proceeded as follows. First, prior to executing the treatment, participants were asked about their headache activity, sleep activity, and medication usage since their last session. This assessment took approximately 5 minutes to complete. Second, the EZPIR headband was secured around the top of the head and the headset was positioned at Fpz (i.e., center of the forehead). Third, the BioEra program was opened. Fourth, the DVD was inserted into the optical disc drive of the notebook computer. Fifth, the BioEra program was started and the play mode of the DVD was set to the continuous play mode for $5 \mathrm{~min}$. After the first $5 \mathrm{~min}$, 
the play mode of the DVD was changed to the auto threshold mode. At this time, participants were instructed to try to maintain a mental state that was simultaneously calm and focused. If the movie paused, the participant was instructed to relax and to focus. The length of time the system remained at the auto threshold mode varied from 10 to 25 minutes and was determined by the session number and the participant's response to the treatment. For the first three sessions, time at the auto threshold mode was limited to $10 \mathrm{~min}$. For the remaining seven sessions, the length of time at the auto threshold mode was limited to $25 \mathrm{~min}$.

Sessions were discontinued for the following reasons: (a) the participant developed a headache, (b) the participant became fatigued, (c) the participant experienced physical or emotional discomfort, (d) rapid fluctuations in the pIR signal were observed, or (e) the pIR signal dropped below the auto threshold level five times. Discontinuation occurred on average two times per participant.

Participants completed the HIT-6 and the MIDAS questionnaire on three occasions: baseline (Pretest), after the sixth treatment session (Midtreatment), and after the tenth treatment session (Posttest). Following completion of data collection, the principal investigator conducted quantitative analyses to assess changes in the impact headache-related disability, attack frequency, and attack severity.

\section{Data Analysis}

Data collected from the demographic questionnaire, HIT-6, and MIDAS questionnaire were entered into a dataset using SPSS (IBM Corp., Armonk, NY; version 21.0). First, descriptive statistics were conducted to explore the frequencies and percentages of the demographic variables among the participants.

Second, a one-way within-subjects multivariate analysis of variance (MANOVA) was conducted to determine whether pIR HEG resulted in a significant multivariate effect. Significant multivariate effects were followed up with corresponding univariate tests. Significant interactions were followed up with pairwise comparisons to determine whether the intervention resulted in a significant change on the relevant dependent measures from Pretest to Midtreatment, Midtreatment to Posttest, and Pretest to Posttest. The headache instruments (i.e., HIT-6 and MIDAS questionnaire) are the repeated measures and pIR HEG is the intervention.

\section{Results}

A one-way MANOVA was conducted to evaluate the sample means for scores on the HIT-6, MIDAS, MIDAS subscale $A$, and MIDAS subscale $B$ at three time points for the 10-week protocol of pIR HEG. The within-subjects factor was the time point and included three levels: pretreatment (baseline), midtreatment (after six treatment sessions), and posttreatment (after 10 treatment sessions). The four dependent variables were HIT-6, MIDAS, MIDAS subscale $A$, and MIDAS subscale $B$ mean scores. Significant differences were found among the three time points on the dependent measures, Wilks's $\Lambda=.45, F(8,114)=7.00, p<.05$. The multivariate $\eta^{2}$ of .33 indicated a strong relationship between time point and the dependent variables. Table 4 contains the means and the standard deviations on the dependent variables for the three time points.

\section{Table 4}

Means and Standard Deviations on HIT-6, MIDAS, MIDAS subscale A, and MIDAS subscale B Scores for the Three Time Points

\begin{tabular}{|c|c|c|c|c|c|c|}
\hline \multirow{2}{*}{$\begin{array}{l}\text { Measure } \\
\text { Mean Scores }\end{array}$} & \multicolumn{2}{|c|}{ Pretest } & \multicolumn{2}{|c|}{ Midtreatment } & \multicolumn{2}{|c|}{ Posttest } \\
\hline & $M$ & $S D$ & $M$ & $S D$ & $M$ & $S D$ \\
\hline HIT-6 & 64.32 & 4.58 & 58.19 & 6.41 & 55.87 & 6.36 \\
\hline MIDAS & 33.36 & 37.26 & 23.84 & 29.08 & 16.32 & 20.79 \\
\hline MIDAS_A & 25.58 & 26.49 & 22.87 & 24.08 & 17.26 & 18.52 \\
\hline MIDAS_B & 6.94 & 1.18 & 5.42 & 1.67 & 5.13 & 1.28 \\
\hline
\end{tabular}

Note. $N=31$. HIT-6 = Headache Impact Test-6; MIDAS = Migraine Disability Assessment; MIDAS_A = Migraine Disability Assessment subscale A; MIDAS_B = Migraine Disability Assessment subscale B. 
Analyses of variance (ANOVA) on each of the dependent variables were conducted as follow-up tests to the MANOVA. The Bonferroni method was used to control for familywise error across the four tests. Therefore, alpha was set at .05 divided by 4 or .0125, for each test. Three of the ANOVAs were significant: the HIT-6 scores, $F(2,60)=24.63, p$ $<.001, \eta^{2}=.45$; the MIDAS scores, $F(1.56,51.59)=$
$8.39, p=.002, \eta^{2}=.22 ;$ and the MIDAS subscale $B$ scores, $F(2,60)=17.33, p<.001, \eta^{2}=.37$. The univariate ANOVA for the MIDAS subscale A scores was nonsignificant, $F(1.38,41.34)=2.30, p=.129$, $\eta^{2}=.07$. Table 5 contains the results of the univariate ANOVAs for the HIT-6, MIDAS, MIDAS subscale $A$, and MIDAS subscale $B$ scores.

\section{Table 5}

One-Way Within-subjects Analyses of Variance for HIT-6, MIDAS, MIDAS subscale A, and MIDAS subscale B Scores

\begin{tabular}{lrrrr}
\hline Measure & \multicolumn{1}{c}{$M S$} & $F$ & $p$ & $\eta^{2}$ \\
\hline HIT-6 & 591.01 & 24.63 & $<.001^{*}$ & .45 \\
MIDAS & 2258.59 & 8.39 & $.002^{*}$ & .22 \\
MIDAS_A & 558.58 & 2.30 & .129 & .07 \\
MIDAS_B & 29.17 & 17.33 & $<.001^{*}$ & .37
\end{tabular}

Note. $N=31 . \eta^{2}=$ effect size. $d f(8,114)$. ${ }^{*}$ alpha set at $p<.0125$ to determine significance.

Post hoc pairwise comparisons for the univariate ANOVAs for the HIT-6, MIDAS, and MIDAS subscale $B$ scores were then conducted to evaluate if there was a difference in HIT-6, MIDAS, and MIDAS subscale $B$ mean scores across the three time points. The Holm's sequential Bonferroni approach (Holm, 1979) was used to control for familywise error across the three tests. Therefore, each pairwise comparison was tested at the alpha level of .0125 divided by 3 , or .004 .

For the HIT-6 scores, significant differences were found among two of the time points: from pretest to midtreatment, and from pretest to posttest. The greatest reduction in HIT-6 scores was from pretest to posttest (8.45 points, $S E=1.46, p<.001, \mathrm{LL}=$ 2.36, UL $=12.94)$. The next greatest reduction in HIT-6 scores was found from pretest to midtreatment (6.13 points, $S E=2.36, p<.001, \mathrm{LL}=2.32, \mathrm{UL}=$ 9.94). However, there was no significant difference in the HIT-6 mean scores from midtreatment to posttest. For the MIDAS scores, significant differences were found at one time point, from pretest to posttest 17.03, $S E=4.42, p=.002, \mathrm{LL}=$ 3.39, UL $=30.67$. No significant differences were found between pretest and midtreatment, or between midtreatment and posttest. For the MIDAS subscale B scores, significant differences were found at two of the time points, from pretest to midtreatment, and from pretest to posttest. The greatest reduction in MIDAS subscale $B$ scores was from pretest to posttest 1.81, SE $=.30, p<.001$, LL $=.87, \mathrm{UL}=2.74$. The next greatest reduction in MIDAS subscale $B$ scores was from pretest to midtreatment, $1.52, \mathrm{SE}=.37, p=.001, \mathrm{LL}=.38$, UL $=2.65$. No significant differences were found between midtreatment and posttest.

The results indicated, in the sample population, a significant reduction in the HIT-6 mean scores (global impact of headache-related disability) from pretest to the midtreatment, and from pretest to posttest following in a 10-week protocol of pIR HEG. Results also indicated a significant reduction in the MIDAS mean scores (functional impact of headacherelated disability) from pretest to posttest. While the MIDAS mean scores changes did not yield significance for pretest to the sixth treatment session, or for the sixth treatment session to posttest, results indicated a significant reduction in the MIDAS subscale $B$ mean scores (severity of attacks) from pretest to midtreatment, and from pretest to posttest. Table 6 presents the 99\% confidence intervals for the pairwise comparisons. 
Table 6

Results of $99 \%$ Confidence Intervals of Pairwise Differences in Mean Changes in HIT-6, MIDAS, and MIDAS subscale B Scores among Three Time Points

\begin{tabular}{llll}
\hline Variable & \multicolumn{1}{c}{$\begin{array}{c}\text { Time } \\
\text { Point }\end{array}$} & \multicolumn{1}{c}{$\begin{array}{c}\text { Time } \\
\text { Point }\end{array}$} & $\begin{array}{c}\text { Mean } \\
\text { Difference }\end{array}$ \\
\hline HIT-6 & & & \\
& Pretest & Midtreatment & $6.13^{*}$ \\
& Pretest & Posttest & $8.45^{*}$ \\
& Midtreatment & Posttest & 2.32 \\
MIDAS & & & \\
& Pretest & Midtreatment & 9.52 \\
& Pretest & Posttest & $17.03^{*}$ \\
& Midtreatment & Posttreatment & 7.52 \\
MIDAS_B & & & \\
& Pretest & Midtreatment & $1.52^{*}$ \\
& Pretest & Posttest & $1.81^{*}$ \\
& Midtreatment & Posttest & 0.29 \\
\hline
\end{tabular}

Note. $N=31 .{ }^{*} p<.004$. Pretest $=$ baseline; Follow-up $=$ after 6 treatment sessions; Posttest $=$ after 10 treatment sessions.

\section{Discussion}

This study found a significant reduction in the global impact of headache-related disability (HIT-6 scores) from pretest to midtreatment, and from posttest. Results indicate a significant reduction in the functional impact of headache-related disability (MIDAS scores) from pretest to posttest. There were no significant differences in attack frequency (MIDAS subscale A scores) across the three time points. However, results indicated a significant reduction in attack severity (MIDAS subscale $B$ scores) from pretest to midtreatment, and from pretest to posttest.

This study represents an important and unique contribution to the literature on nonpharmacologic interventions for the treatment of migraine in a number of respects. First, the current study included a sample that was more homogenous than that in any previously conducted research (cf. Carmen, 2004). Second, treatment was standardized for all subjects to 10 weeks, and limited to pIR HEG alone. Third, this research is among the first to use empirically validated assessments of headache impact (HIT-6, MIDAS Questionnaire) and to include assessments at three time points (baseline, after six treatment sessions, and after 10 treatment sessions).

Like Carmen's case series, this study showed reduction in headache severity. In the current 10week treatment, headache frequency did not decline, in keeping with Carmen's report that frequency of headache occurred for some subjects only months after reduction in pain severity (Carmen, 2004). Whereas Carmen's positive case series provided both pIR HEG, but also other methods such as psychotherapy as applicable, the present study found good results using Carmen's pIR HEG system alone.

Findings from this study should be interpreted with caution because of several limitations. First, this study focused on a sample population with unique characteristics. This sample was comprised of adults with the migraine subtype MWOA and whose baseline HIT-6 scores indicated that headache impact was substantial to very severe. Therefore, it does not reflect the full range of migraine sufferers. A more diverse sample may produce different results which may be more generalizable.

Second, while this study evaluated multiple outcome measures (i.e., the global impact of headacherelated disability, the functional impact of headacherelated disability, the severity of attacks, and the frequency of attacks), consideration could be given to additional outcome measures, including changes in medication usage (e.g., a reduction in medication usage), an assessment of whether participants maintained symptom reduction after completing the treatment, and changes in overall healthcare costs and utilization of doctor visits. It would be beneficial to this population if future studies included these additional research elements. Likewise, this study did not include a placebo or control group. A placebo controlled RCT could significantly enhance the findings of successful outcomes.

It would be beneficial to migraine sufferers if future research included a qualitative component. A qualitative component would provide a more comprehensive picture of the overall impact of migraine on the lives of individual sufferers and humanize a condition that is often treated with derision and disbelief. 


\section{References}

Abramson, J. H., Hopp, C., \& Epstein, L. M. (1980). Migraine and non-migrainous headaches. A community survey in Jerusalem. Journal of Epidemiology and Community Health, 34(3), 188-193. http://dx.doi.org/10.1136/jech.34.3.188

Berg, J., \& Ramadan, N. M. (2006). Societal burden of the headache. In J. Olesen, P. J. Goadsby, N. M. Ramadan, P. Tfelt-Hansen, \& K. M. A. Welch (Eds.), The Headaches (3rd ed., pp. 35-42). Philadelphia, PA: Lippincott, Williams, \& Wilkins.

Bille, B. (1981). Migraine in childhood and its prognosis. Cephalalgia, 1(2), 71-75. http://dx.doi.org/10.1111/j.14682982.1981.tb00012.x

Bjorner, J. B., Kosinski, M., \& Ware, J. E., Jr. (2003a). Using item response theory to calibrate the Headache Impact Test (HIT TM $)$ to the metric of traditional headache scales. Quality of Life Research, 12(8), 981-1002. http://dx.doi.org/10.1023 IA:1026123400242

Bjorner, J. B., Kosinski, M., \& Ware, J. E., Jr. (2003b). Calibration of an item pool for assessing the burden of headaches: An application of item response theory to the Headache Impact Test (HIT $\left.{ }^{\mathrm{TM}}\right)$. Quality of Life Research, 12(8), 913-933. http://dx.doi.org/ 10.1023/A:1026163113446

Blumenfeld, A., \& Tischio, M. (2003). Center of excellence for headache care: Group model at Kaiser Permanente. Headache, 43(5), 431-440. http://dx.doi.org/10.1046/j.15264610.2003.03087.x

Buse, D. C., Rupnow, M. F. T., \& Lipton, R. B. (2009). Assessing and managing all aspects of migraine: Migraine attacks, migraine-related functional impairment, common comorbidities, and quality of life. Mayo Clinic Proceedings, 84(5), 422-435. http://dx.doi.org/10.1016/S00256196(11)60561-2

Carmen, J. A. (2004). Passive Infrared Hemoencephalography: Four years and 100 migraines. Journal of Neurotherapy, 8(3), 23-51. http://dx.doi.org/10.1300/J184v08n03_03

Cutrer, F. M., \& Olesen, J. (2006). Migraines with aura and their subforms. In J. Olesen, P. J. Goadsby, N. M. Ramadan, P. Tfelt-Hansen, \& K. M. A. Welch (Eds.), The Headaches (3rd ed., pp. 407-421). Philadelphia, PA: Lippincott, Williams, \& Wilkins.

Dahlöf, C. G. H., \& Solomon, G. D. (2006). Impact of the headache on the individual and family. In J. Olesen, P. J. Goadsby, N. M. Ramadan, P. Tfelt-Hansen, \& K. M. A. Welch (Eds.), The Headaches (3rd ed., pp. 27-34). Philadelphia, PA: Lippincott, Williams, \& Wilkins.

Dalsgaard-Nielsen, T., Engberg-Pedersen, H., \& Holm, H. E. (1970). Clinical and statistical investigations of the epidemiology of migraine. Danish Medical Bulletin, 17(5), 138-148.

Diamond, S., Bigal, M. E., Silberstein, S., Loder, E., Reed, M., \& Lipton, R. B. (2007). Patterns of diagnosis and acute and preventive treatment for migraine in the United States: Results from the American Migraine Prevalence and Prevention Study. Headache, 47(3), 355-363. http://dx.doi.org/10.1111/j.1526-4610.2006.00631.x

EZPIR system (BioEra version 2.3.109) [Apparatus and software]. (2004). Manlius, NY: Jeff A. Carmen.

Freides, D., \& Aberbach, L. (2005). Exploring hemispheric differences in infrared brain emissions. Journal of Neurotherapy, 8(3), 53-61. http://dx.doi.org/10.1300 IJ184v08n03_04

Gandek, B., Alacoque, J., Uzun, V., Andrew-Hobbs, M., \& Davis, K. (2003). Translating the short-form headache impact test (HIT-6) in 27 countries: Methodological and conceptual issues. Quality of Life Research, 12(8), 975-979. http://dx.doi.org/ 10.1023/A:1026171315263

Harpole, L. H., Samsa, G. P., Jurgelski, A. E., Shipley, J. L., Bernstein, A., \& Matchar, D. B. (2003). Headache management program improves outcome for chronic headache. Headache, 43(7), 715-724. http://dx.doi.org/10.1046/j.1526-4610.2003.03128.x

Headache Classification Subcommittee of the International Headache Society [IHS]. (2004). The International Classification of Headache Disorders [ICHD-II] (2nd ed.). Cephalalgia, 24, Suppl. 1: 9-160. http://dx.doi.org/10.1111/j.1468-2982.2004.00653.x

Heppner, P. P., Kivlighan, D. M., Jr., \& Wampold, B. E. (1999). Quasi-Experimental and Time-Series Designs. Research Design in Counseling (2nd ed., pp. 151-172). Belmont, CA: Wadsworth Publishing Company.

Holm, S. (1979). A simple sequentially rejective multiple test procedure. Scandinavian Journal of Statistics, 6(2), 65-70.

Kosinski, M., Bayliss, M. S., Bjorner, J. B., Ware, J. E., Jr., Garber, W. H., Batenhorst, A., ... Tepper, S. (2003). A sixitem short-form survey for measuring headache impact: The HIT-6 TM. Quality of Life Research, 12(8), 963-974. http://dx.doi.org/10.1023/A:1026119331193

Lemstra, M., Stewart, B., \& Olszynski, W. P. (2002). Effectiveness of multidisciplinary intervention in the treatment of migraine: $\mathrm{A}$ randomized clinical trial. Headache, 42(9), 845-854. http://dx.doi.org/10.1046/j.1526-4610.2002.02202.xLipton, R. B., Bigal, M. E., Diamond, M., Freitag, F., Reed, M. L., \& Stewart, W. F. (2007). Migraine prevalence, disease burden, and the need for preventive therapy. Neurology, 68(5), 343349. http://dx.doi.org/10.1212 /01.wnl.0000252808.97649.21

Lipton, R. B., Scher, A. I., Kolodner, K., Liberman, J., Steiner, T. J., \& Stewart, W. F. (2002). Migraine in the United States: Epidemiology and patterns of health care use. Neurology, 58(6), 885-894. http://dx.doi.org/10.1212/WNL.58.6.885

Lipton, R. B., Stewart, W. F., Diamond, S., Diamond, M. L., \& Reed, M. (2001). Prevalence and burden of migraine in the United States: Data from the American Migraine Study II. Headache, 41(7), 646-657. http://dx.doi.org/10.1046/j.15264610.2001.041007646.x

Lipton, R. B., Stewart, W. F., Sawyer, J., \& Edmeads, J. G. (2001). Clinical utility of an instrument assessing migraine disability: The Migraine Disability Assessment (MIDAS) questionnaire. Headache, 41(9), 854-861. http://dx.doi.org /10.1111/j.1526-4610.2001.01156.x

Magnoux, E., Freeman, M. A., \& Zlotnik, G. (2007). MIDAS and HIT-6 French translation: Reliability and correlation between tests. Cephalalgia, 28(1), 26-34. http://dx.doi.org/10.1111 /j.1468-2982.2007.01461.x

Mathew, N. T., \& Tfelt-Hansen, P. (2006). General and pharmacologic approach to migraine management. In $\mathrm{J}$. Olesen, P. J. Goadsby, N. M. Ramadan, P. Tfelt-Hansen, \& K. M. A. Welch (Eds.), The Headaches (3rd ed., pp. 433440). Philadelphia, PA: Lippincott, Williams, \& Wilkins.

McGrath, P. J., Penzien, D., \& Rains, J. C. (2006). Psychological and behavioral treatments of migraines. In J. Olesen, P. J. Goadsby, N. M. Ramadan, P. Tfelt-Hansen, \& K. M. A. Welch (Eds.), The Headaches (3rd ed., pp. 441-448). Philadelphia, PA: Lippincott, Williams, \& Wilkins.

Merriam-Webster. (2005). Merriam-Webster's collegiate dictionary (11th ed.). Springfield, MA: Author.

Nachit-Ouinekh, F., Dartigues, J.-F., Henry, P., Becg, J.-P. Chastan, G., Lemaire, N., \& El Hasnaoui, A. (2005). Use of the headache impact test (HIT-6) in general practice: Relationship with quality of life and severity. European Journal of Neurology, 12(3), 189-193. http://dx.doi.org/10.1111/j.1468-1331.2004.00934.x

Nikiforow, R. (1981). Headache in a random sample of 200 persons: A clinical study of a population in northern Finland. Cephalalgia, 1(2), 99-107. http://dx.doi.org/10.1111/j.14682982.1981.tb00016.x

Olesen, J., \& Dodick, D. W. (2006). The history and examination of headache patients. In J. Olesen, N. M. Ramadan, P. Tfelt- 
Hansen, \& K. M. A. Welch (Eds.), The Headaches (3rd ed., pp. 43-54). Philadelphia, PA: Lippincott, Williams, \& Wilkins.

Olesen, J., \& Goadsby, P. J. (2006). The migraines: Introduction. In J. Olesen, P. J. Goadsby, N. M. Ramadan, P. TfeltHansen, \& K. M. A. Welch (Eds.), The Headaches (3rd ed., pp. 231-233). Philadelphia, PA: Lippincott, Williams, \& Wilkins.

Parsons, A. A. (2006). Nitric Oxide. In J. Olesen, P. J. Goadsby, N. M. Ramadan, P. Tfelt-Hansen, \& K. M. A. Welch (Eds.), The Headaches (3rd ed., pp. 151-158). Philadelphia, PA: Lippincott, Williams, \& Wilkins.

Patel, N. V., Bigal, M. E., Kolodner, K. B., Leotta, C., Lafata, J. E., \& Lipton, R. B. (2004). Prevalence and impact of migraine and probable migraine in a health plan. Neurology, 63(8), 1432-1438. 101.WNL.0000142044.22226.54

Penzien, D. B., Rains, J. C., Lipchik, G. L., Nicholson, R. A., Lake, A. E., \& Hursey, K. G. (2005). Future directions in behavioral headache research: Applications for an evolving health care environment, Headache, 45(5), 526-534.

Pliszka, S. (2003). Neuroscience for the mental health clinician. New York, NY: The Guilford Press.

Sacks, O. (1992). Migraine: Revised and Expanded. New York, NY: Vintage Books.

Sillanpää, M., \& Anttila, P. (1996). Increasing prevalence of headache in 7-year-old schoolchildren. Headache, 36(8), 466-470. http://dx.doi.org/10.1046/j.15264610.1996.3608466.x

Stang, P. E., \& Osterhaus, J. T. (1993). Impact of migraine in the United States: Data from the National Health Interview Survey. Headache, 33(1), 29-35. http://dx.doi.org/10.1111 /j.1526-4610.1993.hed3301029.x

Steiner, T. J., Scher, A. I., Stewart, W. F., Kolodner, K., Liberman, J., \& Lipton, R. B. (2003). The prevalence and disability burden of adult migraine in England and their relationships to age, gender and ethnicity. Cephalalgia, 23(7), 519-527. http://dx.doi.org/10.1046/j.1468-2982.2003.00568.x

Stewart, W. F., Lipton, R. B., Celentano, D. D., \& Reed, M. L. (1992). Prevalence of migraine headache in the United States. Relation to age, income, race, and other sociodemographic factors. The Journal of the American Medical Association, 267(1), 64-69.

Stewart, W. F., Lipton, R. B., \& Kolodner, K. (2003). Migraine Disability Assessment (MIDAS) score: Relation to headache frequency, pain intensity, and headache symptoms.
Headache, 43(3), 258-265. http://dx.doi.org/10.1046/j.15264610.2003.03050.x

Stewart, W. F., Lipton, R. B., Kolodner, K. B., Liberman, J. N., \& Sawyer J. (1999). Reliability of the migraine disability assessment score in a population-based sample of headache sufferers. Cephalalgia, 19(2), 107-114.

Stewart, W. F., Lipton, R. B., Kolodner, K. B., Sawyer, J., Lee, C., \& Liberman, J. N. (2000). Validity of the Migraine Disability Assessment (MIDAS) score in comparison to a diary-based measure in a population sample of migraine sufferers. Pain, $88(1), \quad 41-52 . \quad$ http://dx.doi.org/10.1016/S03043959(00)00305-5

Stewart, W. F., Lipton, R. B., Whyte, J., Dowson, A., Kolodner, K. B., Liberman, J. N., \& Sawyer, J. (1999). An international study to assess reliability of the Migraine Disability Assessment (MIDAS) score. Neurology, 53(5), 988-994. http://dx.doi.org/10.1212/WNL.53.5.988

Stewart, W. F., Shechter, A., \& Rasmussen, B. K. (1994). Migraine prevalence: A review of population-based studies. Neurology, 44(6), Suppl. 4: S17-23.

Stovner, L. J., \& Scher, A. I. (2006). Epidemiology of headache. In J. Olesen, P. J. Goadsby, N. M. Ramadan, P. Tfelt-Hansen, \& K. M. A. Welch (Eds.), The Headaches (3rd ed., pp. 17-25). Philadelphia, PA: Lippincott, Williams, \& Wilkins.

Tfelt-Hansen, P. (2006). Prioritizing acute pharmacotherapy of migraines. In J. Olesen, P. J. Goadsby, N. M. Ramadan, P. Tfelt-Hansen, \& K. M. A. Welch (Eds.), The Headaches (3rd ed., pp. 515-517). Philadelphia, PA: Lippincott, Williams, \& Wilkins.

Toomim, H., \& Carmen, J. (2009). Hemoencephalography: Photon-based blood flow neurofeedback. In T. H. Budzynski, J. R. Evans, \& A. Abarbunel (Eds.), Introduction to quantitative EEG and neurofeedback: Advanced theory and applications (2nd ed., pp. 169-194). New York, NY: Elsevier.

Zagami, A. S., \& Bahra, A. (2006). Symptomatology of migraines without aura. In J. Olesen, P. J. Goadsby, N. M. Ramadan, P. Tfelt-Hansen, \& K. M. A. Welch (Eds.), The Headaches (3rd ed., pp. 399-405). Philadelphia, PA: Lippincott, Williams, \& Wilkins.

Received: April 17, 2016

Accepted: June 4, 2016

Published: June 21, 2016 